\title{
A Microthread Technique for Studying the Viability of Microbes in a Simulated Airborne State
}

\author{
By K. R. MAY AND H. A. DRUETT \\ Microbiological Research Establishment, Porton, Wiltshire
}

(Accepted for publication I4 October 1967)

\begin{abstract}
SUMMAR Y
A technique is described in which airborne micro-organisms are captured on ultra-fine spider threads. In this state the organisms may be subjected to any environment of interest for extended periods of time, during which their loss of viability may be assayed. Experiments are described which show that the loss of viability is quantitatively similar to that in the true airborne state, provided that the microthread and aerosol environments are identical and that certain other precautions are observed.
\end{abstract}

\section{INTRODUCTION}

For some diseases it is well established that infection can be acquired by inhalation of airborne particles which consist of, or contain, viable bacteria or viruses. In the study of these infections it is obviously desirable to be able to investigate such factors as: (a) the length of time in which micro-organisms can remain viable in the airborne environment; $(b)$ the extent to which loss of viability is affected by the bulk or physical nature of the particle; $(c)$ the effect of sterilizing agents in the air, such as ultraviolet radiation and vapours toxic to the microbes. These aspects can be studied to some extent in small rooms or specially constructed chambers, but drift, fall-out, loss on walls, etc., is usually such as to limit severely the time period of such experiments. The effect of radiation from a sterilizing source on airborne particles in rooms, chambers, etc., is particularly difficult to measure quantitively because random air movement continually changes the distance of the particles from the radiation source. The rotating-drum apparatus (Goldberg et al. 1958) can be used to hold small particles of artificially generated aerosols in the airborne state for very long periods, but the survival of micro-organisms in these tightly enclosed conditions may not be the same as in inhabited environments. Therefore the results are of uncertain value in public health applications.

The extent to which fungal spores and pollens remain viable is also difficult to study because, when captured from the open air, their age is usually unknown and their size is such that in any enclosure they rapidly fall out from the aerosol.

In an effort to overcome these difficulties we have studied the survival of microorganisms when they are supported on ultra-fine threads stretched across a small metal frame. This system gives a form of 'captive aerosol' with which, for example, it is easy to subject particles to accurately measured dosages of sterilizing vapours or radiation for any length of time and in any condition of humidity and temperature. By combining the viable particles with an inert tracer such as a stable bacterial spore or a 
radioactive substance the loss of viability of the particles with time can be studied in detail, though their infectivity as airborne particles cannot be tested.

For our purposes we consider the basic requirements for microthreads to be:

(i) Their diameter should be as small as possible, and in any case smaller than that of the smallest viable particle. Access to the particle of vapours, radiation, humidity change, etc., should then not be seriously impeded by the presence of the supporting threads.

(ii) They should be biologically inactive so that there is no toxic or protective action at the point of contact of particle with thread.

(iii) They should be sterile and free from surface contamination before use.

(iv) They should be easy to handle and to wind round their holding frames in a standardized manner.

(v) When wound on the frames they should be resistant to shock, shaking and to the forces of air movement.

(vi) They should be in plentiful supply.

(vii) A standardized method of winding should give a constant length of thread on every frame so that the number of particles caught shall be predictable.

Commercially or experimentally made fibres fail to meet one or more of these requirements, particularly (i) and (iv). Kordyum \& Bobchenko (I959) studied the growth of micro-organisms on fine glass threads $(50 \mu)$ diameter but we do not know of any means of obtaining uniform and regular supplies of glass threads to our requirement for fineness ( $<\mathrm{I} \mu$ diameter) in a form convenient for handling. Dessens (1949) used spider threads to collect and study atmospheric haze particles. He allowed spiders to crawl over metal frames and pointed out that some of the threads were as fine as $0.01 \mu$.

We now routinely use the 'safety-line' threads released by recently hatched specimens of those spiders which spin orb webs. The safety-line threads are produced by the spider in its normal escape activity when it falls from a vigorously tapped rod or frame. These threads are about $0.5 \mu$ in diameter; but the spider also produces, in a random and unpredictable manner, side threads which are too fine to be seen under the high-power microscope, so that they can only be detected when particles adhere to them. Presumably in nature these side threads act as wind-blown anchors; in the present work they caused the number of particles caught from a flowing aerosol of known concentration to be unpredictable, except to within a factor of 2 or 3 . In all other respects the fine spider threads appear to fulfil requirements (i)-(vi) perfectly. Also, the weight of the spider, which is exactly right for the thread it produces, gives a constant winding tension for all turns.

\section{APPARATUS AND METHOD}

Frames for microthreads. The frames used for supporting the microthreads were designed so that they are convenient $(a)$ to handle when winding on the threads, $(b)$ to expose in any desired situation, $(c)$ to immerse in sampling fluid when removing the organisms for assay. A frame is shown in Fig. I A. The handle, $7 \cdot 5 \mathrm{~cm}$. (3 inch) long, is made from 13-gauge stainless steel, as are the two cross-pieces. These crosspieces are $22 \mathrm{~mm}$. ( $\frac{7}{8}$ inch) long and are joined by the two side members made from $5.5 \mathrm{~cm}$. ( $2 \frac{1}{8}$ inch) lengths of 19-gauge stainless steel. The outer edges of these side 
members are $1 \cdot 9 \mathrm{~cm}$. ( $\frac{3}{4}$ inch) apart. All joints are silver soldered and all frames are assembled on a jig so that all are identical. A useful degree of protection to the microthreads when wound round the side members is afforded by the cross-pieces, which are thicker than the side members and project beyond the latter at the corners (see Fig. I A).

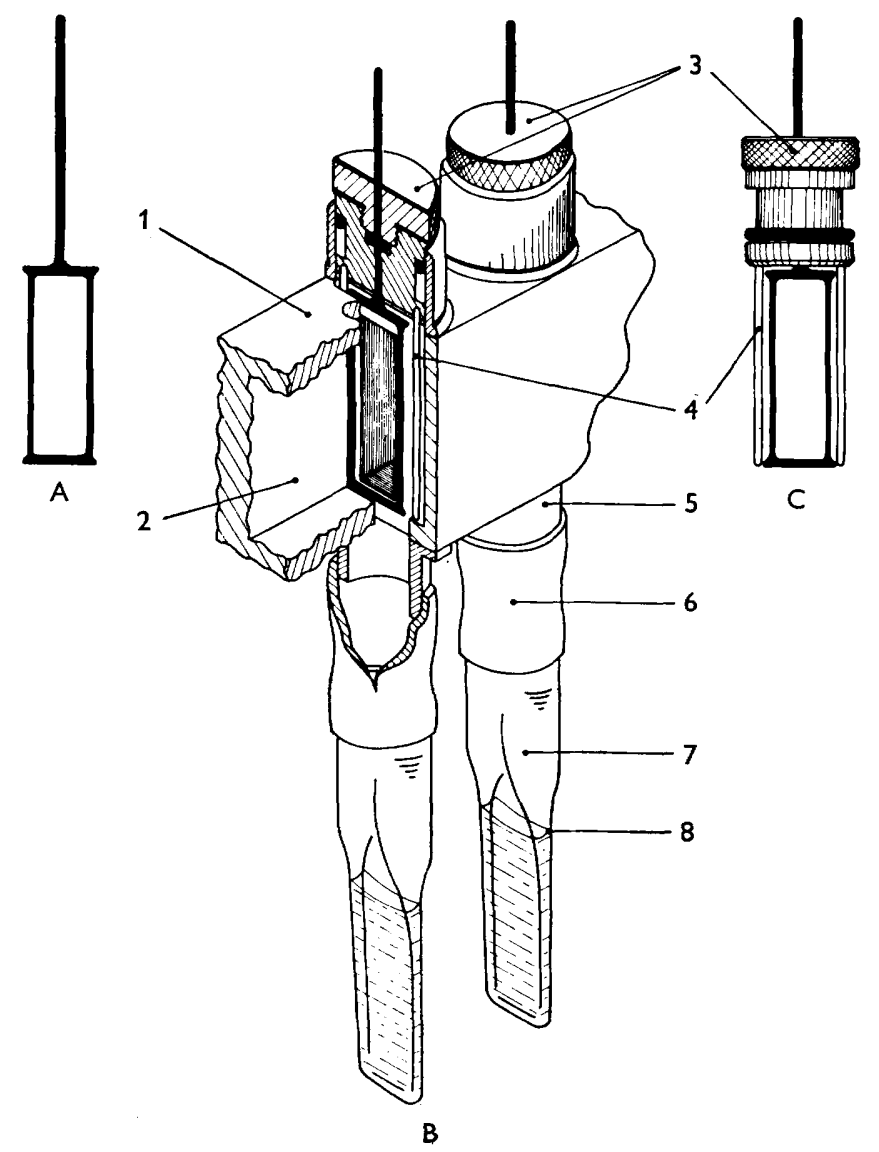

Fig. I. A, Stainless steel frame. B, Isometric cut-away sketch of 'sow' tube showing positioning of frames and sampling cells. $\mathrm{C}$, Frame mounted in removable, O-ring sealed cap.

Spiders. As a source of microthreads we usually employ newly hatched or young specimens (bodies not greater than $3 \mathrm{~mm}$; $\frac{1}{8}$ inch) of Araneus diademata or of Zygiella$x$-notata which are the two commonest orb spinners. The latter can be artifically reared by feeding on Drosophila, to obtain a continuous supply for most of the year. There is no doubt that many other species of web-spinners would be equally satisfactory, but spiders which do not spin web snares are of no value.

Method of winding spider threads on the frames. To wind a frame, a spider is placed on it (a wire loop is a useful transferring tool) and the frame, held horizontally, is given a sharp tap. The spider drops on its safety line which is then wound round the frame by spinning the handle the required number of turns. Normally we use $50 \mathrm{com}-$ plete turns (100 lateral threads) spacing the threads equally along the frames but only to within $\frac{1}{4}$ inch of the cross-pieces (Pl. I, fig. 2). The winding process may also be done 
by holding the handle in a chuck which is mounted on a lead screw with a pitch of I $\mathrm{mm}$. so that, on hand rotation, the frame advances I mm. per turn, with each turn indicated on a counter. When a spider of about $3 \mathrm{~mm}$. ( $\frac{1}{8}$ inch) body is used for this process and the room air is still, very perfectly wound frames can readily be produced. The threads are too fine to be visible in transmitted light but may be readily seen in Tyndall illumination at certain angles. Very close inspection makes possible the rejection of frames which carry an excessive number of the ultra-fine cross-threads, should this be desired. These frames would capture more particles than their neighbours, which may have fewer or no cross-threads. Plate I, fig. I, shows a photomicrograph of two main threads and a single cross-thread, the existence of which is only revealed in transmitted light by the presence of the micro-organisms on it. While a frame is being wound, the spider must not be allowed to climb back onto it or the spider will at once tangle up the threads which have been already wound. A single spider in good health will provide enough thread before it tires to wind from two to eight frames, according to the size of the spider. Spiders larger than about $3 \mathrm{~mm}$. $\left(\frac{1}{8} \mathrm{inch}\right)$ in body size move too quickly and actively to be readily handled and the thread diameter increases in proportion.

Spider threads wound in this way will withstand air velocities well in excess of $20 \mathrm{miles} / \mathrm{hr}(32 \mathrm{~km}$. $/ \mathrm{hr})$ without breaking, their strength relative to their size being extremely high. The silk threads are not ejected by the spider, they are drawn out from the spinnarets and thread formation from the viscous fluid is said to be a process of molecular rearrangement of the amino acids therein and not of drying, oxidation or other chemical process (Savory, 1952).

Frames are washed and sterilized before winding and the threads as produced by the spider are sterile. Provided that wound frames are stored in a closed container, stocks of them may be stored for many weeks without deterioration before use. Contaminated wound frames may be sterilized by ultraviolet radiation or by heating at $60^{\circ}$ for $\mathrm{I} \mathrm{hr}$.

Equipment for capturing and sampling aerosol particles on the microthreads. To deposit aerosol particles on the microthreads a tube called a 'sow' is employed. A cutaway isometric sketch of a small section of a sow is shown in Fig. I B. The rectangular body of the sow tube (I) is built from brass and the internal rectangular channel (2) through which the aerosol passes is $2 \times \frac{1}{2}$ inch $(5 \times \mathrm{I} \cdot 3 \mathrm{~cm}$.). Slots, cut right through the sow body (I) from the top to bottom allow just enough room for a frame to pass right through. To load the sow with frames, the handle of each frame is first pushed through the holding cap (3), a side elevation of which is shown in Fig. I C and a section in Fig. I B. This holding cap has an internal O-ring which seals and grips the handle of the frame. On the external shaft of the holding cap is an O-ring, as shown, which provides a rolling seal when the cap is pushed into its socket on top of the sow.

When the holding cap is pushed home in the sow it holds the frame in the position shown in Fig. I B. The metalwork of the frame is completely recessed within the slot in the walls of the sow so that the aerosol, when passing through the rectangular channel (2), only encounters the microthreads in its direct passage. In fact, owing to some eddies within the slot recesses, about $\mathrm{I}-2 \%$ of the number of particles intercepted by the microthreads may lodge on the frames (see P1. I, fig. 2). The holding caps have two side-prongs (4) which serve as protectors to the frames and as guides when the cap is pushed home in the sow. 
Below each frame station is the tube (5). A short length of thin-walled rubber tubing (6) connects tube (5) to the glass (or plastic) sampling cell (7). These sampling cells are attached as shown in Fig. I B when it is desired to perform an experiment on the survival of the micro-organisms within the enclosure of the sow tube. In such an experiment a screw-clip (not shown in Fig. I B) seals off the tube (6). At suitable time intervals frames can be withdrawn from the sow without opening it to the air by fully opening the screw clip and pushing the handle of the frame down through the holding cap (3) by means of a ramrod of the same diameter as the handle. The frame drops into the cell (7) and at the same time the ramrod maintains the integrity of the seal within (3) as it passes through the small internal O-ring. The screw clip is re-tightened and the cell (7) is withdrawn.

The sampling cells (7) are formed over a mandrel to the shape shown and are normally filled with $4 \mathrm{ml}$. of sampling fluid. The meniscus height (8) is such that when a frame is dropped home its shoulder or upper cross-piece is above the meniscus. A vigorous up-and-down agitation of the frame handle moves the lower cross-piece up and down in the narrow channel, so giving a violent stirring action within the sampling fluid. This removes micro-organisms from the microthreads and thoroughly mixes the suspension for assay. There is no splashing as only the vertical side members of the frame penetrate the liquid surface.

Sows of the Fig. I B pattern have been made with six frame stations. The ends of the rectangular body have flanges so that coned end-tubes may be bolted on. These cones taper to a $\mathrm{I}$-inch $(2.54 \mathrm{~cm}$.) diameter tube, a convenient size for the induction of aerosols. With coned pieces removed, two sows may be bolted together in series. In another form of sow, used solely for depositing particles on the microthreads before removal of the frames to exposure sites, the lower tubes (5) are dispensed with, and a second row of sockets for the caps (3) is built into the intermediate positions. In this way, $I_{3}$ frames may be charged within the same length as needed for 6 frames in the Fig. I B form.

Methods of generating aerosols. In the laboratory artificial aerosols for passage through the sow may be generated by any convenient device, from liquid suspensions or powdered material. For generating aerosols of very small particle size we routinely use a Collison spray system similar to that described by Henderson (1952) but with the addition of humidity and temperature control. For aerosols of large uniformly sized particles the wind-tunnel system of Druett \& May (1952), which embodies a spinning-top sprayer, is employed. The vibrating-reed apparatus of Wolf (I96I) is also useful for depositing perfectly uniform particles on the threads. With Wolf's device the generated droplets may be allowed to fall directly onto the microthreads before evaporation. This avoids the use of the sow but the number of droplets caught is only very roughly predictable. 'Natural' aerosols may be drawn through the sow for capture on the threads, provided that their concentration is high enough to give an adequate number of viable organisms for assay. A sneeze aerosol could be captured in this way. Particle size selection may be incorporated when desired by fitting a simple impaction system of the required performance to the sow intake hole. Details of suitable impactor design parameters are given for example by May (I967). 


\section{Capture efficiency of threads}

Small particles generated from a liquid suspension readily adhere to a microthread after touching it. The well-known van der Waals forces between small particles no doubt play a part, as do surface tension forces at the points of contact, where a minute liquid film condenses in the zone of high curvature (Cross \& Picknett, 1964). When an unevaporated droplet is intercepted by the threads, its residue after drying will be bonded firmly round the thread. Thus, fine aerosol particles, when intercepted by the threads will stay in place in spite of subsequent wind forces or mechanical shock, yet are readily removed and dispersed by the standard procedure in the sampling cells, as already described. However, liberties may not be taken with particles dangerous to health. An initial shake off or blow off of a very small proportion of deposited particles can be demonstrated in certain circumstances and in free air there is always the possibility that a fly, floating feather, etc., might carry off some of the threads.

In very dry conditions and with larger particles adherence to the spider threads is much less satisfactory. Pollens and fungal spores, for example, with their hard dry rough surfaces are very easily removed from the threads by wind or shock. To study the viability of these in the 'captive aerosol' state it may be necessary to use, for example, Terylene threads, which can be obtained down to I5 $\mu$ diameter, a size which is still quite small as compared to some pollens and fungal spores. Such threads could be dipped, after winding on the frames, into a solution which would leave a thin adhesive film on the threads. If it were acceptable to spray the pollens or spores of interest from a liquid suspension, a small proportion of inert adhesive could be incorporated in the liquid, and the particles would then adhere firmly to any type of thread. The very fine spider threads we have described do not have sufficient absolute strength to withstand dipping into an adhesive solution. Electrostatic forces of attraction might also be effective with pollens and spores.

When particles adhere to a thread on contact, the effective 'capture cross-section' of a frame is the product of the total length of thread exposed to the aerosol and the sum of the diameters of the particle and thread. Hence the fraction of particles of a given size removed from the passing aerosol by any one frame is $n l(D+d) / h l$, where, in consistent units, $n=$ number of threads across the frame, $D$ and $d=$ diameters of aerosol particle and thread, $h$ and $l=$ height and width of sow channel. The number of organisms caught on the frame is $N=n(D+d) C V t / h$, where $C=$ total number of organisms per unit volume of air, in the particles of diameter $D, V=$ volumetric flow rate and $t=$ time of exposure.

As $n(D+d) / h$, the fraction of a passing aerosol caught, is very small successive frames are exposed to only slightly decreasing dosages. If the required parameters are known the above calculation is sufficiently accurate for assay dilution values to be estimated, but the variable number of ultra-fine cross-threads, together with certain anomalies of flow within the sow, cause variations in the catch from frame to frame which in extreme cases can exceed $4: I$. A tracer incorporated with the decaying particles nullifies these variations and should therefore be used when possible. When a tracer cannot be used it is necessary to pool the samples from several frames at one time, having rejected before use any frames seen to have excessive fine cross-threads. 


\section{Methods of exposure of frames}

After depositing an adequate number of organisms (usually at least 2000) on the microthreads they may be exposed to the environment of interest in any convenient way. For example, the effect of toxic vapours may be studied by flowing the gas mixture slowly through a sow, with a clean-air sow as a control. To do this two complete sows are set up in series with clean filtered air drawn through at, say, 10 1./min. and the toxic vapour is introduced at the mid-point junction of the sows. Any increased decay rate in the downstream sow as compared to that in the upstream sow is then due to the introduced vapour. The effect of flow velocity will be discussed later.

For exposure in rooms, holding-caps ((3) in Fig. I) with their frames still in place may be inverted and the cap stood on a level surface with the frame handle pushed half-way through the cap. Alternatively a low table-like framework, with holes bored through the level surface, may be used. The holding caps rest on the holes with the microthread frame projecting beneath. This arrangement allows full air circulation but gives some physical protection to the threads.

To study the effects of sunlight on micro-organisms a number of small lightweight counterbalanced wind-vanes were constructed. Each holds a single frame, inverted in respect to Fig. I A. A small polythene windscreen is mounted on the horizontal shaft of the vane just in front of the frame to give the threads protection from the direct force of the wind, dust particles, insects, etc. The polythene is partially transparent to all solar wavelengths in the visible and near ultraviolet.

Whatever the exposure situation, the standard sampling cells have been used for assay.

\section{Measurement of very low degrees of viability}

As already mentioned, the number of small particles lodging on the metal sides of a frame is about $\mathrm{I}-2 \%$ of those caught on 100 threads across the frame (Pl. I, fig. 2). It has been found that micro-organisms in contact with the stainless steel will, in general, survive better than those fully exposed on the threads. Therefore measurements of viability have low significance when the viability has decreased to about I \% of its original level. To overcome this we have used two-pronged forks made from thin stainless-steel wire. The prongs will just pass down inside the side members of the metal frame and when coated with an inert adhesive remove only the microthreads with their attached organisms. These may then be dropped into the standard sampling cells. Reasonably consistent estimates of viability can be obtained in this way at levels below $\mathbf{I} \%$ of the initial viability.

\section{Validity of the technique}

Before the microthread technique could be established as a useful tool it had to be shown that survival of micro-organisms supported on the threads resembled that of the particles in the true airborne state in the same environment. The range of experiments in which such a comparison can be made is limited because the main feature of the microthread technique is that it can be used to study viability where other methods cannot. We were able to make comparison tests in the following three situations where the airborne micro-organisms could be assayed by the liquid impinger technique, which is well-established and considered to be reliable. (I) Survival in small enclosures. 
Survival of true aerosols in the Goldberg rotating drum was compared to survival on the microthreads held both in a closed sow and in a drum. (2) Survival of an aerosol sprayed into a room compared to survival on microthreads in a similar room. (3) Survival of a range of particle sizes under sterilizing radiation (sunlight). The experiments were designed to ensure that the aerosol particles in the airborne state and on the threads came from the same source and were identical in all respects.

The test organism was a strain of Escherichia coli known to be robust in the aerosol state. Into the stock suspension was mixed a proportion of the tracer, spores of Bacillus subtilis var. niger, which are highly resistant to the airborne state and which grow into colonies at about the same rate as $E$. coli on the same media. Sampling cells and impingers were filled with phosphate buffer $(\mathrm{pH} 7)$ containing Manucol in M-sucrose solution; serial dilutions of the samplers were made in the same fluid. Plating was on tryptone agar. After incubation, differential counts of the two contrasting types of colony were made on each plate.

(I) Survival in small enclosures. We considered that if attachment to a microthread were to cause the survival of a micro-organism to be different from that in the true airborne state, then the difference would be more obvious for single-bacterium particles than for particles containing many bacteria, where mutual protection between the organisms might be expected. Single bacteria are nearer to the diameter of the threads and have a large fraction of their surface area in contact with the thread, so that they would be more subject to any adverse influence of the thread. Therefore these first comparisons were made with single-bacterium aerosols from the modified Henderson apparatus. This type of aerosol is in any case the most suitable for holding in the Goldberg rotating drum.

Aerosol was led from the spray mixing tube through the drum and sow in parallel. Drum and sow were then sealed off and held in a constant-temperature environment Samples from the drum were taken at intervals with the 'raised impinger' (May \& Harper 1957), and frames were dropped from the sow at the same intervals.

Comparisons at $65^{\circ} \mathrm{F}\left(17.2^{\circ} \mathrm{C}\right)$ and $95 \% \mathrm{RH}$ are shown in Fig. 2 and at $60^{\circ} \mathrm{F}$ $\left(15.5^{\circ} \mathrm{C}\right)$ and $22 \% \mathrm{RH}$ in Fig. 3. Both show a close agreement between the two methods. In Fig. 3 the triangles show results obtained without a tracer by finding the initial Escherichia coli recovery from 3 pooled frames and expressing successive $E$. coli recoveries from similar pooled samples as percentages of the initial number. The curve shows that it is possible to dispense with a tracer, given an adequate number of frames to assess and provided that the organisms survive the stress of aerosolization to give an initial viability close to $100 \%$.

A typical comparison made at $68^{\circ} \mathrm{F}\left(20^{\circ} \mathrm{C}\right), 50 \% \mathrm{RH}$ is shown in Fig. 4 . In this series of comparisons a persistently higher viability was obtained from the threads, the reason for which is unknown. The difference between the curves is, however, not important in comparison with the much higher rates of die-away which are usual when micro-organisms are being studied in larger enclosures such as a room where the air is in movement and contains sterilizing components (see Fig. 5). One difficulty encountered in long-term holding experiments is that very slight traces of a toxic vapour in a holding vessel will show a very pronounced unfavourable effect on the maintenance of viability in that vessel. Therefore it is essential to ensure that holding vessels used for control experiments are scrupulously clean inside.

In other tests, survival in the drum against survival in the sow was compared as 


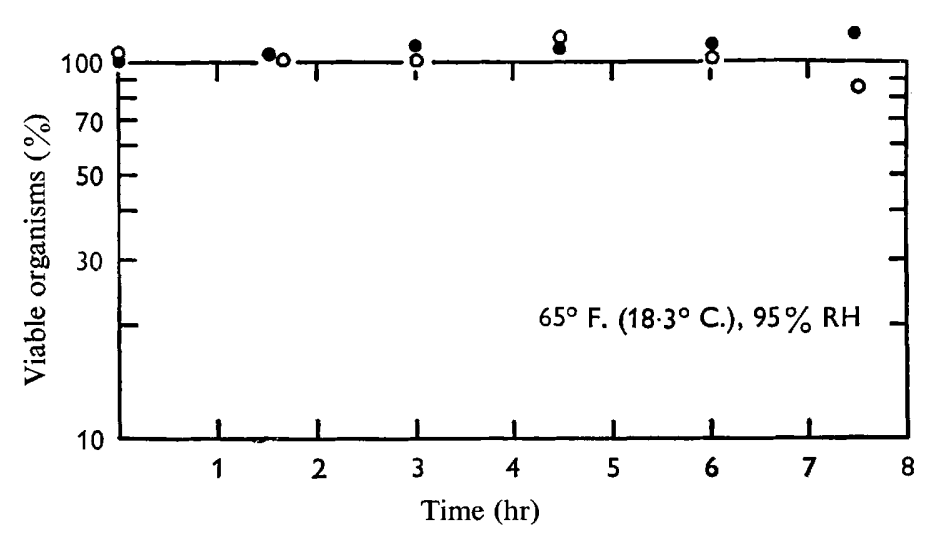

Fig. 2

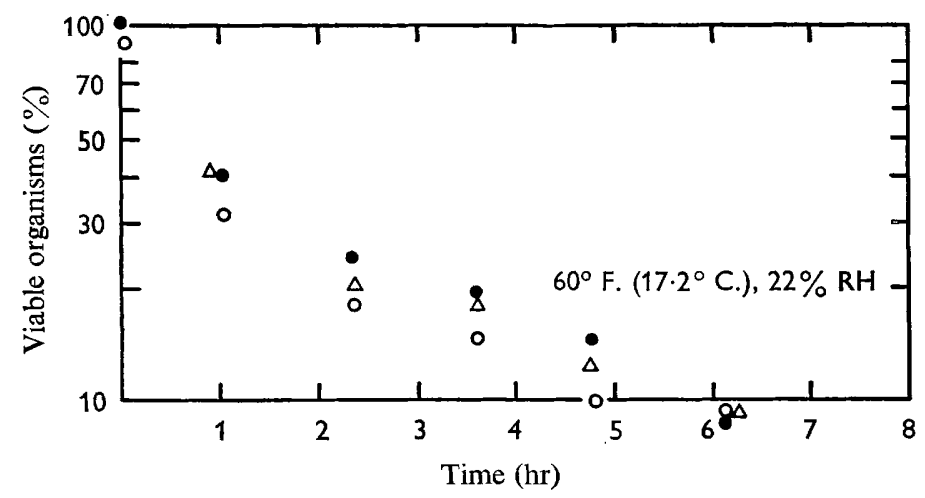

Fig. 3

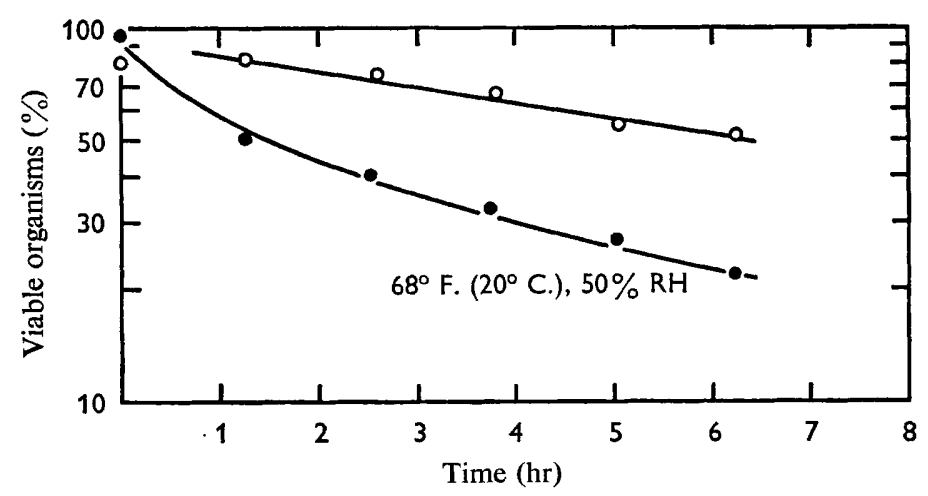

Fig. 4

Fig. 2. Comparison of survival of Escherichia coli I 62 single-bacterium particles at high humidity; $\mathrm{O}$, on microthreads; 0 , aerosol particles in rotating drum. Each point is the mean from three replicate samples.

Fig. 3. As Fig. 2 but at low humidity. $\triangle$, Escherichia coli viabilities estimated without use of tracer.

Fig. 4. As Fig. 2 but at intermediate humidity. 
above, but in addition survival on threads held in an otherwise empty aerosol drum was compared to that in the adjacent sow. After $2 \mathrm{I} \mathrm{hr}$ at $69^{\circ} \mathrm{F}\left(20.5^{\circ} \mathrm{C}\right) 63 \% \mathrm{RH}$ the measured viabilities in all three systems were in the range $4-10 \%$. It was concluded that, provided that full precautions were taken in cleanliness and in establishing identical environmental conditions, there is no serious systematic difference between survival of particles in the airborne state in the drum and the same particles held on the microthreads.

(2) Survival in a room. A sealed chamber, $\mathrm{I} 6 \times 8 \times \mathrm{I} 2 \mathrm{ft}$., was filled by an aerosol from the standard suspension by a remotely controlled pneumatic spray. The chamber was fanned for a short period to allow thorough mixing and evaporation of the spray droplets. The final particle diameters ranged from $6 \mu$ downwards. Twenty litres of the aerosol was then drawn through a sow via an access port in I min. and an impinger

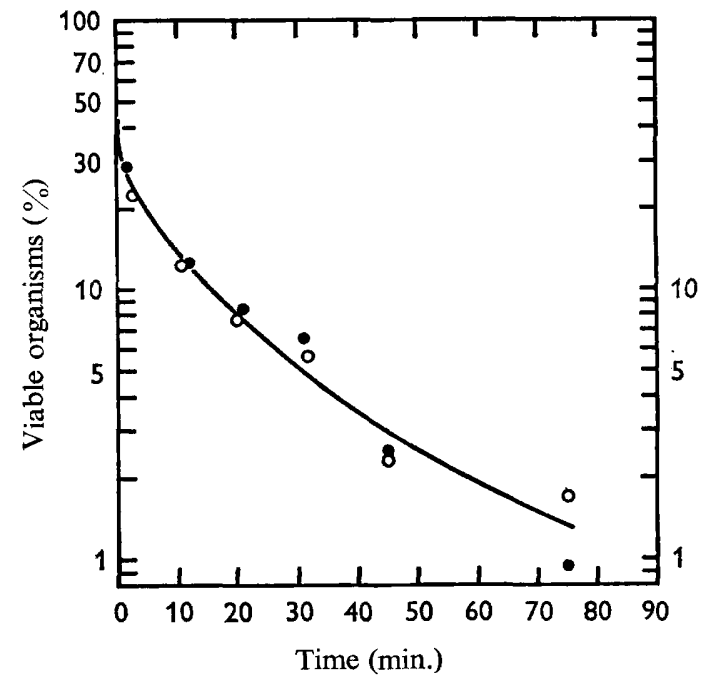

Fig. 5

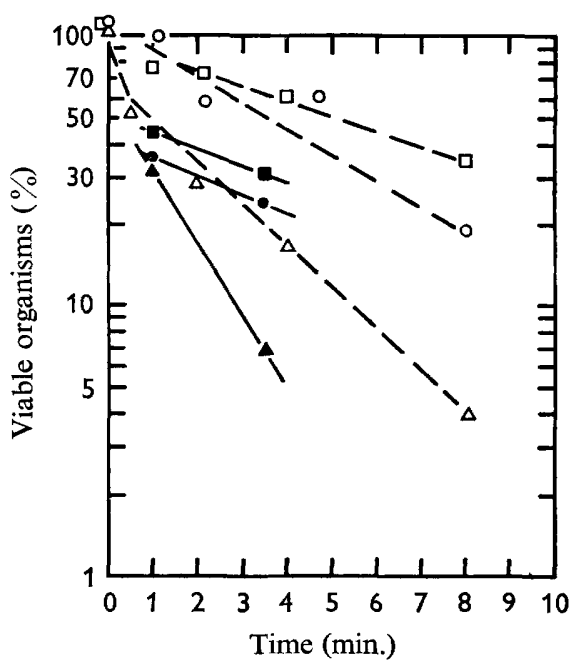

Fig. 6

Fig. 5. Comparison of survival of Escherichia coli 162 particles in a room; $O$, on microthreads; 0 , aerosol particles sampled by impinger.

Fig. 6. Comparison of survival of sprayed particles of Escherichia coli 162 in sunlight, in three particle-size ranges. On microthreads; $\triangle$, particles $<3 \mu$; $\bigcirc$, particles $3-6 \mu$; $\square$, particles $>6 \mu$. Airborne particles; $\boldsymbol{\Lambda}$, particles $\langle 3 \mu$; $\boldsymbol{0}$, particles $3-6 \mu ; \mathbf{\square}$, particles $>6 \mu$.

sample was taken at the same time. The sow was then removed to an adjoining chamber, which was a replica of the first, the frames were removed and exposed on a table. Both chambers were fanned at frequent intervals, and impinger and frame samples were taken simultaneously from each chamber during the experiment. Three experiments gave very similar results, one of which is shown in Fig. 5. The agreement between the two methods was very close.

The rate of loss of viability in Fig. 5 is very high compared to the result of Fig. 4, which was obtained in tightly enclosed conditions in rather similar temperature and humidity. Some toxic component in the air of the chambers may have been present on this occasion, as it is possible to obtain prolonged survival of this strain of Escherichia coli in a room (cf. Fig. 7). 
(3) Survival in sterilizing radiation. Tests on a three-stage glass impinger were described by May (1966). This device separates an aerosol into particle-size fractions of $>6 \mu, 6-3 \mu$, and $<3 \mu$, and permits viability determinations to be made with each fraction. In these tests a pneumatic spray of a suspension of Escherichia coli and Bacillus subtilis var. niger was released into the air and it was possible to sample some of the aerosol after it had travelled downwind for 3-4 min. To compare the microthread technique in these conditions, frames in three sows were charged with particles of $9 \mu, 4.5 \mu$ and I $-2 \mu$, respectively, to represent the mid-points of the ranges in the 3-stage impinger. The frames were then taken to a flat roof on a fine summer day

Table I. Percentage viability of airborne Escherichia coli

\begin{tabular}{rcrcc} 
& & \multicolumn{3}{c}{ Particle size range } \\
\cline { 3 - 5 } Sampler I & Time airborne & $>6 \mu$ & $6-3 \mu$ & $<3 \mu$ \\
Sampler 2 & I min. & 55 & 38 & 35 \\
Average & I min. & 35 & 36 & 3 I \\
Sampler I & I min. & 45 & 37 & 33 \\
Sampler 2 & $3 \frac{1}{2}$ min. & 30 & 28 & 10 \\
Average & $3 \frac{1}{2}$ min. & 32 & 21 & 4 \\
& $3 \frac{1}{2}$ min. & 31 & $24 \cdot 5$ & 7
\end{tabular}

Table 2. Decay rates $(\% /$ min. $)$ of airborne and threadborne Escherichia coli

\begin{tabular}{lrcc} 
& \multicolumn{3}{c}{ Average particle size } \\
\cline { 2 - 4 } Impinger samples & $9 \mu$ & $4.5 \mu$ & I-2 $\mu$ \\
Thread samples & I4 & 16 & 63 \\
& 13 & 22 & 36
\end{tabular}

with about 5/10 cloud and were mounted in the 'Vane' holding and exposure device at about the same time as the pneumatic spray cloud was released nearby. The suspensions for the airborne cloud and for the threadborne particles came from the same stock. The I-2 $\mu$ particles were generated in a Henderson apparatus at the ambient temperature and humidity and the larger particles were obtained from the Druett \& May wind-tunnel apparatus. Because of the practical difficulties of the tests it was possible to obtain no more than duplicate 3-stage impinger samples at two time-intervals in the airborne state, namely after I min. and $3.5 \mathrm{~min}$. average travel. The percentage of $E$. coli viable in the impinger samples is given in Table I (taken from May, 1966, table 2, p. 567, runs 4 and 5). The averages from Table I are plotted in Fig. 6 for comparison with the microthread experiment done at the same time. The impinger data are obviously scanty but there is general agreement with other figures given by May for similar atmospheric conditions. Straight lines drawn through the plotted points in Fig. 6. appear to start from different origins, perhaps because the pneumatic spraying into the air gave an initial kill of some of the E. coli. Slopes of these lines are given in Table 2 , in terms of percentage loss of viability among the $E$. coli cells per minute. Table 2 indicates agreement between the 'captive' and true aerosol viability measurements in that both show rapid and rather similar decay under the powerful sterilizing action of the diffuse or direct insolation and that both show the smallest particles dying off more rapidly than the larger ones. Col. 3 suggests that the smallest 
particles survived rather better on the threads. Thread and particle were of comparable dimension here, and there may well have been some radiation shielding. Further experiments were made, but are not reported because comparisons were not made simultaneously. There was no suggestion from the latter that the microthread results had any gross systematic difference from the airborne cloud results.

\section{Precautions required by the technique}

Air velocity over the threads. An airborne particle falls through air at its terminal velocity, which is, for example, $0.0035 \mathrm{I} \mathrm{cm}$. $/ \mathrm{sec}$. for a I $\mu$ particle and $0.309 \mathrm{~cm} . / \mathrm{sec}$. for a io $\mu$ particle. These velocities are extremely small compared to normal air movements past stationary microthreads. To study the effect of air velocity, an I8-inch radius arm was set up inside an open-topped drum and could be electrically rotated to give a tip speed of $20 \mathrm{miles} / \mathrm{hr}$. $(32 \mathrm{~km}$./hr). Frames were mounted in a special holder on the tip of the arm and a similar holder half-way along the arm gave ro miles/ hr (I $6 \mathrm{~km}$./hr). Further frames could be held very close to the axis in almost stagnant air. Frames were mounted edge-on to the air flow to minimise collection of the room dust by the microthreads.

The result of an experiment in a room containing clean, stable, almost still, air favourable to the survival of the Escherichia coli (in single-bacterium particles) is shown in Fig. 7. There is a very clear relation between death rate and air velocity, the death rates at 20 , Io and I mile/hr $(32,16, \mathrm{I} \cdot 6 \mathrm{~km}$./hr) being approximately 3, 2 and $1 \% / \mathrm{min}$. Figure 8 shows the result of another experiment, which was carried out in an identical fashion to that in Fig. 7 except that the air contained a sterilizing component and was itself in fairly rapid movement above the drum. In these conditions there was little difference in death rate. After the first $30 \mathrm{~min}$. the viability decreased at about $17 \% / \mathrm{min}$. at the 20 miles $/ \mathrm{hr}(32 \mathrm{~km}$. $/ \mathrm{hr})$ velocity, but the three sets of points (i) at Io miles $/ \mathrm{hr}$ (I6 km./hr), (ii) in the nearly still air at the centre of the shielding drum, and (iii) from the stationary frames outside the drum, all gave about $10 \% / \mathrm{min}$.

The reason for this difference in velocity dependence in favourable and hostile atmospheres is not understood. The effect has been well established by several experiments replicating those of Figs. 7 and 8. In another series of experiments air was drawn at a steady rate through a tube whose cross-section area decreased in steps. Frames could be exposed at different points down this tube so that all were in the same air but experienced air velocities ranging from 0.2 to $23 \mathrm{miles} / \mathrm{hr}(0.32-36 \mathrm{~km}$. $/ \mathrm{hr})$ according to their site of exposure. Comparisons between favourable (filtered) air and hostile (containing sterilizing components) air closely matched those of Figs. 7 and 8 in all respects. This dependence upon air velocity when rates of decay are low is clearly a difficulty with the microthread technique. To overcome or minimize this we are careful to expose microthreads to air which is in the same state of movement as would obtain in the airborne cloud which is being simulated. This was the situation in Figs. 2-6, where satisfactory agreement with the comparison situations was obtained.

Frame-to-frame variations. Our experience is that replicate assays (using a tracer) from a number of identically exposed frames usually show wider scatter in viability determination than replicate impinger samples from an aerosol. Single or duplicate frame assays at single points in time may therefore give less smooth decay curves than is desirable. The simplicity of the technique will usually allow several frames to be assayed per point to obtain greater precision if required. Sampling and assay 
procedures must always be carried out in an identical, standardized, manner. Studies with radioactively labelled organisms have shown that the proportions of test and tracer organisms caught on the threads is the same as in the passing aerosol (provided that the particles are similar in size and in surface characteristics) so that assay variations beyond statistical expectations arise between dropping the frames into the sampling fluid and counting the incubated colonies.

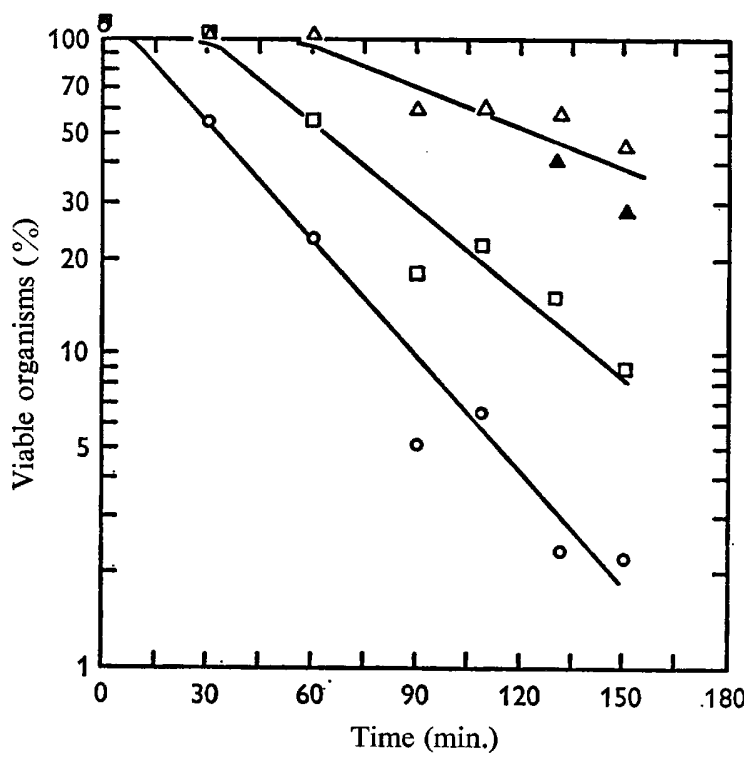

Fig. 7

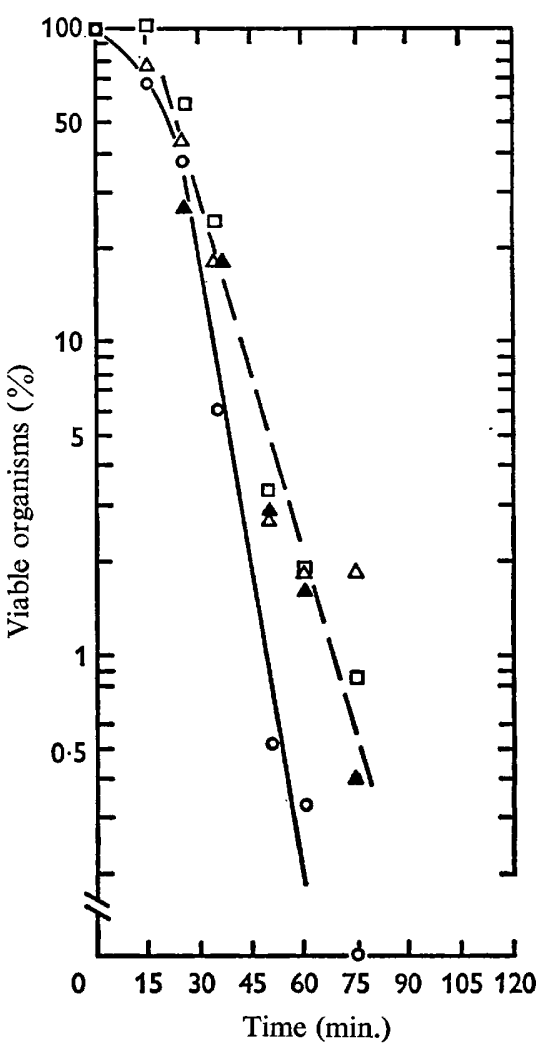

Fig. 8

Fig. 7. Effect of air velocity on survival of Escherichia coli 162 single-bacterium particles in whirling-arm experiment in a room containing clean air; $O, 20$ miles $/ \mathrm{hr}(32 \mathrm{~km} . / \mathrm{hr}) ; \square$, $10 \mathrm{miles} / \mathrm{hr}(\mathrm{I} 6 \mathrm{~km} . / \mathrm{hr}) ; \triangle, \sim$ o miles $/ \mathrm{hr}$ at axis of $\mathrm{arm} ; \boldsymbol{\Delta} \sim \mathrm{o} \mathrm{miles} / \mathrm{hr}$ on bench in room.

Fig. 8. Effect of velocity of air containing a sterilizing component. Whirling-arm experiment. Symbols as in Fig. 7 .

\section{Conclusions}

The comparisons described in this paper together with 4 years experience with the microthread technique lead us to conclude that it is a useful tool in the study of air borne microbes, provided that the proper precautions are observed. Of particular value is its versatility, in that there is, within reason, no limit to the length of time, ranges of atmospheric conditions, radiation intensity, etc., in which the airborne state can be simulated. Moreover, organisms such as pathogens can be studied in actual living spaces instead of having to be confined, in the aerosol state, to unrealistic sealed vessels. Extensive studies have been made of the sterilizing effect of various vapours and atmospheres; these will be published later. 


\section{REFERENCES}

Cross, N. L. \& PicknetT, R. G. (1964). Mechanism of corrosion by fuel impurities. Marchwood Conference 1963, p. 383. London: Butterworth.

Dessens, H. (1949). The use of spider's threads in the study of condensation nuclei. Q. Jl R. met. Soc. $75,23$.

Druetr, H. A. \& MAY, K. R. (1952). A wind tunnel for the study of airborne infections. J. Hyg., Camb. 50, 69.

Goldberg, L. J., Watkins, H. M. S., Boerke, E. E. \& Chatigny, M. A. (1958). The use of a rotating drum for the study of aerosols over extended periods of time. Am.J. Hyg. 68, 85.

Henderson, D. W. (1952). An apparatus for the study of airborne infection. J. Hyg., Camb. 5o, 53.

Kordyum, V. A. \& BoBChenKo, E. S. (I959). Air as a habitat for micro-organisms. Microbiology 28, 215.

MAY, K. R. \& HARPER, G. J. (I957). The efficiency of various liquid impinger samplers in bacterial aerosols. Br. J. ind. Med. 14, 287.

MAY, K. R. (I966). Multi-stage liquid impinger. Bact. Rev. 30, 559.

May, K. R. (1967). Physical aspects of sampling airborne microbes. Symp. Soc. gen. Microbiol. $\mathbf{I 7}, 60$.

SAVORY, T. H. (1952). The Spider's Web. London: Warne.

WoLF, W. R. (196I). Study of the vibrating reed in the production of small droplets and solid particles of uniform size. Rev. Scient. Instrum. 32, I 124.

\section{EXPLANATION OF PLATE}

Fig. I. Photomicrograph of dried particles, from a sprayed bacterial suspension, adhering to microthreads.

Fig. 2. Autoradiograph of frame after exposure in a sow to an aerosol of particles containing ${ }^{131}$. Note faint deposition on metal work and heavy deposition on the microthreads. 
Journal of General Microbiology, Vol. 5I, No. 3

Plate I
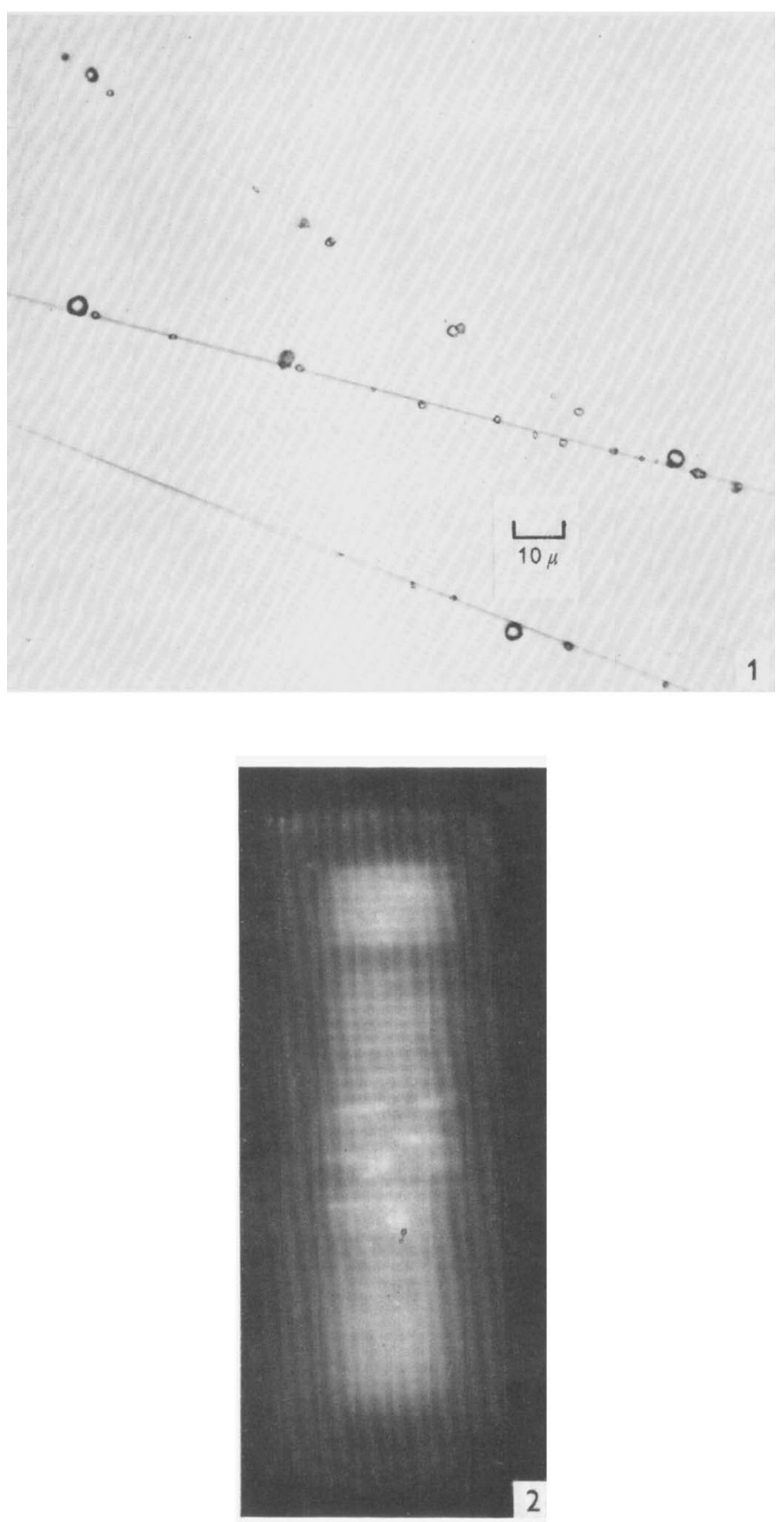